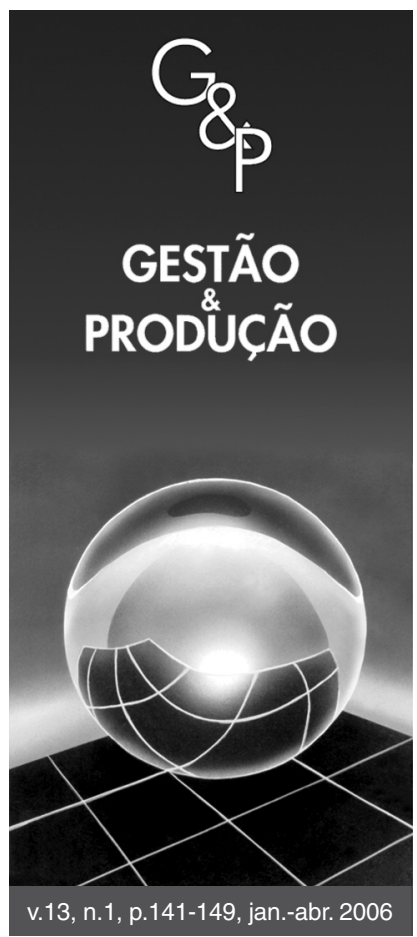

\title{
ATITUDES DE ALUNOS E PROFESSORES COM RELAÇÃO A CURSOS DE MESTRADO EM ENGENHARIA DE PRODUÇÃO A DISTÂNCIA
}

\author{
Andrea Valéria Steil \\ Instituto Virtual de Estudos Avançados - VIAS, Rod SC 401, Km 1, Parqtec Alfa, \\ Bairro João Paulo, CEP 88030-000, Florianópolis, SC, \\ e-mail: andrea@vias.org.br \\ Ricardo Miranda Barcia \\ Instituto Virtual de Estudos Avançados - VIAS, Rod SC 401, Km 1, Parqtec Alfa, \\ Bairro João Paulo, CEP 88030-000, Florianópolis, SC \\ Programa de Pós-Graduação em Engenharia e Gestão do Conhecimento, \\ Universidade Federal de Santa Catarina, \\ e-mail: rbarcia@vias.org.br
}

Recebido em 06/4/2005

Aceito em 27/3/2006

Resumo

Este artigo resulta de uma pesquisa que se propôs a analisar dados de um relatório realizado em uma instituição de ensino superior brasileira, que teve como foco a avaliação do processo de desenvolvimento de cursos de mestrado em Engenharia de Produção na modalidade de educação a distância (EAD). A experiência deste mestrado a distância é única no Brasil e seu modelo pedagógico recebeu um prêmio de qualidade pela Associação Brasileira de Educação a Distância (ABED). O artigo focaliza três aspectos avaliados no relatório acerca das atitudes dos professores e dos alunos em relação à modalidade educacional a distância: A) as atitudes de professores e de alunos em relação à interação proporcionada; $B$ ) as atitudes dos alunos em relação ao papel do professor; $e$ C) as expectativas dos alunos pelo curso. A abordagem teórica utilizada foi a teoria das atitudes, o conceito de esquemas representativos e os construtos referentes às atitudes diante de diferentes aspectos da educação a distância. Trata-se de um estudo exploratório e de natureza quantitativa. A partir destes dados, discute-se a influência destas atitudes no tempo de conclusão de curso do aluno. Os sujeitos da pesquisa são 471 alunos e 30 professores. Os dados foram coletados por meio de questionário. Os resultados indicam que as atitudes de alunos e de professores foram predominantemente positivas em relação aos aspectos investigados. Identificou-se que na modalidade educacional em questão o tempo de titulação dos alunos ficou muito próximo ao ideal sugerido pela CAPES/MEC. Sugere-se que tal resultado possa estar relacionado com as atitudes positivas dos alunos com relação aos seus cursos e ao modelo pedagógico e à estrutura de apoio oferecidos ao aluno pelo curso. Diferentemente dos resultados de algumas pesquisas referentes ao papel das atitudes diante da educação a distância, os resultados deste estudo também sugerem que atitudes positivas (ou negativas) diante da educação a distância estão relacionadas tanto com o modelo pedagógico adotado quanto com a tecnologia utilizada. Os resultados deste estudo abrem espaço para a investigação mais detalhada a respeito do papel das atitudes dos alunos em cursos de mestrado a distância em Engenharia de Produção.

Palavras-chave: educação a distância, atitudes, engenharia de produção.

\section{Introdução}

Há crescente interesse na compreensão das atitudes de estudantes e de professores com relação à adoção de inovações educacionais, identificado pelo número de estudos em desenvolvimento nessa área (Ocker, 2001; Berge, 2002). Inman et al. (1999), por exemplo, identifi- caram que um dos fatores que contribuem para a pequena taxa da adoção da educação a distância (EAD) pelas instituições de ensino superior pode estar relacionada à existência de atitudes negativas quanto a essa inovação educacional. 
Um fator que influencia as atitudes diz respeito ao papel do professor e dos alunos na educação a distância, que não assume a mesma configuração que a da sala de aula tradicional, desafiando os esquemas de representação vigentes. Os esquemas são organizações cognitivas que representam o conhecimento sobre um conceito desenvolvido pela experiência passada, as quais influenciam a forma pela qual novas informações e situações são organizadas (Fiske e Taylor, 1984). Como os esquemas representam uma relação prototípica, eles conduzirão o indivíduo a comparar e a julgar todas as suas experiências educacionais futuras com relação ao seu esquema.

O esquema estabelecido com relação à situação de ensino formal baseia-se na presença dos professores e dos alunos em um mesmo espaço e tempo. Como conseqüência, assume-se que existe uma necessidade básica da manutenção dessa configuração para que a aprendizagem ocorra (Inman et al., 1999). Em função deste aspecto, as configurações educacionais que diferem desse esquema podem ser vistas com apreensão e ceticismo.

Considerando-se tais aspectos, este artigo tem o objetivo de colaborar com as discussões sobre as atitudes de alunos e de professores participantes de cursos na modalidade educacional a distância. Para tanto, são utilizados dados de um relatório realizado por uma instituição de ensino superior localizada no sul do Brasil que teve como foco a avaliação do processo de desenvolvimento de cursos de mestrado em Engenharia de Produção na modalidade educacional a distância. Os dados enfocam três dos aspectos avaliados no relatório acerca das atitudes dos professores e alunos em relação à modalidade educacional a distância: A) as atitudes de professores e alunos em relação à interação proporcionada; B) as atitudes dos alunos em relação ao papel do professor; e C) as expectativas dos alunos pelo curso.

$\mathrm{O}$ artigo ainda reflete sobre a possível influencia destes três aspectos no tempo que o aluno leva para concluir o curso de mestrado, uma vez que alguns estudos apontam a vigência do fenômeno da inexistência de diferença significativa (non significance difference phenomenon) entre os resultados da aprendizagem das modalidades educacionais a distância e presencial (Philipps e Merisotis, 1999).

Este artigo procura contribuir com as discussões teóricas acerca das atitudes de professores e de alunos no âmbito da educação a distância, mais precisamente nos cursos de mestrado a distância em Engenharia de Produção. Os resultados deste artigo poderão ser utilizados por instituições de ensino superior que planejam iniciar um processo de virtualização de suas atividades educacionais de pós-graduação stricto sensu.

Para o alcance de seu objetivo, o artigo está estruturado como se segue. As primeiras seções tratam dos fun- damentos teóricos: a definição e as funções das atitudes, assim como os resultados de estudos que identificaram as atitudes de alunos e professores diante de inovações educacionais. Logo após, a metodologia é apresentada, com o detalhamento dos participantes da pesquisa e dos procedimentos utilizados. Os resultados são apresentados em seguida. Por fim, são discutidos os resultados e são apresentadas as considerações finais.

\section{Definição e funções das atitudes}

A atitude é considerada um dos principais construtos das ciências comportamentais e sociais. Existe consenso teórico sobre a compreensão das atitudes como disposições mentais para avaliar um objeto psicológico, expressas em dimensões de atributos, como bom/mau, agradável/desagradável, etc. (Ajzen, 2001; Wood, 2000). Há indícios crescentes de que a avaliação dirigida a um objeto surge imediatamente, sem esforço consciente. Essas avaliações são ativadas automaticamente mesmo quando os julgamentos avaliativos não são esperados ou solicitados (Bargh e Chartrand, 1999).

As atitudes são influenciadas simultaneamente pela cognição e pelo afeto. O grau de influência desses dois elementos difere entre os indivíduos na determinação das atitudes dirigidas a diferentes objetos. Apesar desse aspecto, quando a cognição e os sentimentos com relação a um objeto possuem valências opostas, a influência dos sentimentos tende a predominar (Lavine et al., 1998). De forma complementar, a informação negativa tem maior impacto no desenvolvimento das atitudes, quando comparada com informações muito positivas. Há experimentos que indicam que existe maior atividade cognitiva e melhor memória para as palavras negativas do que para as positivas (Ajzen, 2001).

As funções das atitudes estão relacionadas ao aumento da adaptação do indivíduo ao ambiente. Em termos específicos, essas funções podem ser: a) de expressão de valores; b) de conhecimento; c) de defesa egóica; d) de ajustamento social; e e) de utilização (ou utilitária). Em consonância com essas funções, as atitudes possuem um efeito tendencioso sobre os julgamentos e a memória. Os indivíduos tendem a aceitar materiais e idéias que são consistentes com a atitude existente e a não levar em consideração o que está em conflito com ela (Ajzen, 2001; Havice, 1999).

\section{Atitudes com relação à educação a distância}

Há evidências teóricas de que as atitudes dos estudantes com relação à educação a distância são indicadores tão importantes quanto o seu desempenho para a identificação da eficácia dos cursos e dos programas realizados a 
distância (Valenta et al., 2001). Em função desse aspecto, a necessidade de se explorarem as atitudes dos alunos quando da implementação de tecnologias educacionais é sugerida (Ocker, 2001). De acordo com Havice (1999), as atitudes influenciam a motivação para aprender e ajudam a delinear as ações humanas, incluindo a aceitação de mensagens educacionais.

A literatura relacionada às atitudes dos estudantes ante seus cursos sugere que a mídia utilizada como método de instrução não tem influência nas atitudes (Havice, 1999). Apesar desse indício, os resultados das pesquisas sobre os efeitos da educação a distância nas atitudes de estudantes e professores diante de diferentes cursos ainda não são conclusivos (McGreal, 1994).

Por exemplo, um estudo experimental comparou se havia ou não diferença nas atitudes de estudantes universitários com relação a um sistema integrado de mídia, quando comparado com o método tradicional de palestras. Os resultados indicaram que não houve diferença significativa nas atitudes em direção ao curso nos grupos experimental e de controle (Havice, 1999). De forma diferenciada, em um estudo com professores universitários dos Estados Unidos, Inman et al. (1999) verificaram que os professores pesquisados possuem atitudes conflitantes sobre a educação a distância. Eles demonstram vontade de participar de cursos a distância, mas possuem ainda dúvidas sobre a sua qualidade. Ainda, quanto mais experiente é o professor na sala de aula tradicional, menos satisfeito ele está, em princípio, como professor na metodologia a distância.

Akerlind e Trevitt (1999) realizaram uma revisão dos fatores que induzem os estudantes a resistir à aprendizagem mediada por tecnologia. Os referidos autores concluíram que, em termos gerais, quanto mais satisfeitos os estudantes estão com as suas experiências de aprendizagem tradicionais, sem o uso de tecnologias, menos eles estarão preparados para aceitar métodos de aprendizagens não familiares. Para esses estudantes, a imposição externa para o uso de métodos tecnológicos, quando os estudantes possuem pouco conhecimento sobre o tema, geralmente produz sentimentos de desconforto e de ansiedade, de perda de controle pessoal e de medo de: a) aprender menos; e b) não saber que elementos do tópico em estudo devem ser estudados em profundidade.

Um outro estudo sobre as atitudes em face da utilização de sistemas tecnológicos de auto-aprendizagem, realizado com alunos de graduação em Marketing, indicou que as atitudes dos alunos dependem da motivação individual para o alcance de sucesso na profissão e/ou da curiosidade do estudante e do seu envolvimento com o curso (Bennet e Kottasz, 2001).

Em um estudo realizado na comunidade européia, com 847 alunos matriculados em cursos de graduação em di- ferentes países, verificou-se que a maioria dos alunos apresentou uma atitude positiva diante das oportunidades oferecidas pela tecnologia no processo educacional. Apesar desse aspecto, houve um número significativo de alunos que considerou que as tecnologias podem também adicionar um grau de complexidade no processo educacional, que pode ser maior do que a sua efetiva capacidade de absorção (Dondi et al., 2004).

Nesta seção, foram descritos os principais estudos sobre o tema, que formam a base teórica para o delineamento desta pesquisa. Entretanto, apesar do número crescente de estudos relacionados às atitudes diante da educação a distância, este ainda não se configura em um corpo teórico unificado, especialmente quando são investigadas conjuntamente as atitudes de alunos e professores.

\section{Metodologia}

O estudo propõe a análise de parte dos dados de um relatório realizado em uma instituição de ensino superior brasileira que teve como foco a avaliação do processo de desenvolvimento de cursos de mestrado em Engenharia de Produção na modalidade educacional a distância (EAD). O relatório, de uma forma geral, descreve as atitudes, o funcionamento, os sistemas de apoio, os recursos didáticos e o aparato tecnológico dos cursos de mestrado a distância em Engenharia de Produção desenvolvidos pela respectiva instituição. Cabe ressaltar que a experiência deste mestrado acadêmico a distância é única no Brasil e seu modelo pedagógico recebeu um prêmio de qualidade pela Associação Brasileira de Educação a Distância (ABED).

Trata-se de um estudo exploratório, de natureza quantitativa. A amostra foi constituída por 471 alunos e 30 professores integrantes dos cursos de mestrado a distância em Engenharia de Produção. Os alunos encontravam-se em período de créditos.

A coleta, o levantamento e análise dos dados ocorreram no período de setembro a outubro de 2000. Os alunos responderam ao instrumento durante o horário das aulas. Já os professores, receberam os questionários em mãos, os quais foram posteriormente recolhidos pelos pesquisadores. Todas as respostas dadas aos questionários foram anônimas. Apesar do tempo decorrido da experiência, em função da natureza unívoca do mestrado presencial virtual analisado, considera-se importante o registro e a divulgação de seus resultados na comunidade acadêmica.

$\mathrm{O}$ instrumento utilizado para coletar os dados sobre a atitude de alunos e professores foi um questionário elaborado especificamente para este fim, pelos responsáveis pelo desenvolvimento do relatório. As assertivas do questionário foram desenvolvidas a partir da análise de diferentes instrumentos de aferição de atitudes (Perez e White, 1985; Singhanayak e Hooper, 1998). O instru- 
mento não foi alvo de validação estatística, entretanto, pela natureza inovadora da experiência, considera-se o potencial teor de reflexão resultante da utilização do instrumento.

No que diz respeito às atitudes de alunos e professores, o questionário investigou três aspectos. São eles: A) as atitudes de alunos e professores com relação à interação proporcionada pelo modelo de educação a distância vigente; B) atitudes dos alunos com relação ao corpo docente; e C) as expectativas dos alunos frente ao curso de mestrado EAD.

Para o item A), atitudes de alunos e professores com relação à interação proporcionada pelo modelo EAD vigente, foram elaboradas três assertivas: 1) o modelo EAD propicia interação entre alunos; 2) o modelo EAD propicia interação entre aluno e orientador; e 3) o modelo EAD propicia interação entre aluno e professor. Para o item B), atitudes dos alunos com relação ao corpo docente foram delineadas quatro assertivas: 1) tempo de resposta às dúvidas via ambiente de aprendizagem on-line; 2) ambiente de abertura e respeito; 3 ) relação entre conteúdo e prática; e 4) estímulo ao uso do ambiente de aprendizagem on-line. $\mathrm{O}$ item $\mathrm{C}$ ), dirigido especificamente aos alunos, investigou se o curso de mestrado estava atendendo às suas expectativas. Para todas as assertivas, foram apresentadas quatro opções de resposta: 1) pouco; 2) regular; 3) satisfatório; 4) bom; e 5) ótimo. Antes de se aplicarem os questionários, foi adotado o procedimento de validação semântica.

\section{Apresentação e discussão dos resultados}

Antes da exposição dos resultados da escala atitudinal, alguns dados referentes ao modelo pedagógico do curso e ao perfil dos alunos e professores participantes do estudo serão apresentados.

\subsection{Modelo pedagógico do curso}

Apesar de a expressão "educação a distância" ser utilizada, na época da pesquisa, os cursos de mestrado a distância da referida universidade apresentavam como modelo educacional o "presencial virtual", pois fazia uso da videoconferência interativa para a efetivação das aulas, por meio da qual efetiva-se o diálogo imediato, com áudio e vídeo em tempo real, entre professores e alunos. Segundo Decreto n 2494 (MEC, 1998), a educação a distância é uma modalidade de ensino que possibilita a auto-aprendizagem, com a mediação de recursos didáticos sistematicamente organizados, apresentados em diferentes suportes de informação, utilizados isoladamente ou combinados, e veiculados pelos diversos meios de comunicação.

Diferentemente da noção de educação a distância baseada em auto-aprendizagem, neste modelo o aluno "não assistia" a aulas previamente formatadas ou gravadas em vídeo e respondia questões em sua apostila. Aluno e professor se viam mutuamente, com retorno integral de áudio, imagens e dados, em tempo real. Por isso não havia "veiculação" de vídeo e material didático e sim a interação direta, por meio da telepresença entre os participantes da aula (professores, alunos e monitores).

A estrutura de apoio ao aluno era formada pelo coordenador, pelos professores das disciplinas, por professores orientadores, tutores de orientação, um coordenador de orientação e um monitor. O tutor de orientação é um aluno de doutorado do professor orientador em fase final de desenvolvimento de sua tese, que auxilia o mestrando no processo de definição do problema de pesquisa, na revisão de literatura, na metodologia de pesquisa e nas discussões dos resultados, juntamente com o professor orientador. $\mathrm{O}$ coordenador de orientação é um professor-pesquisador da universidade com reconhecida experiência em pesquisas. A coordenação de orientação acompanha o desenvolvimento das dissertações mediante o contato sistemático com todos os alunos e seus respectivos orientadores. O monitor é um especialista em educação a distância que tem como meta interagir sistematicamente com os alunos para garantir os seguintes pontos: manter a motivação dos alunos pelo curso; auxiliar nas trocas colaborativas entre os alunos; e informar sobre os processos de desenvolvimento do curso.

Essa estrutura de apoio ao desenvolvimento das dissertações pode ter minimizado a identificação de diferenças fundamentais entre a situação prototípica da educação presencial para a experiência atual da realização de um mestrado a distância com as configurações descritas. Os alunos matriculados nos cursos presenciais virtuais tinham ciência de que a estrutura elaborada para o seu acompanhamento era formada por uma quantidade maior de profissionais especializados do que tinham à disposição os seus colegas matriculados nos mesmos cursos de mestrado presenciais (UFSC, 2001).

\subsection{Perfil dos alunos de mestrado a distância da universidade}

Com relação à faixa etária, a maior concentração de alunos possuía entre 30 e 39 anos (38,90\%), seguida da faixa entre 40 e 49 anos $(31,69 \%)$ e entre 20 e 29 anos $(21,39 \%)$. A maior parte dos alunos era do sexo masculino $(64,47 \%)$.

Como os cursos de mestrado em Engenharia de Produção no Brasil atraem egressos de diferentes áreas do conhecimento, buscou-se verificar esse comportamento nos cursos em questão. O maior contingente de alunos provém de cursos de administração $(36,8 \%)$, seguido das engenharias $(14,4 \%)$, ciências contábeis $(11,6 \%)$, psicologia $(7,8 \%)$ e pedagogia $(6,4 \%)$. Os principais fatores motivacionais para o ingresso nos cursos foram assim 
hierarquizados pela amostra pesquisada: a) atualização profissional $(23,13 \%)$; b) aprimoramento/realização pessoal $(14,30 \%)$; c) desafio pessoal $(14,30 \%)$; d) base teórica para aplicar na profissão $(14,03 \%)$; e) interesse pela área $(11,56 \%)$; f) necessidade de titulação $(9,77 \%)$; g) interesse em ingressar em uma instituição de ensino $(6,78 \%)$; e h) outros $(6,13 \%)$.

Com relação ao perfil, também foram identificadas as experiências anteriores dos alunos com as tecnologias da videoconferência e Internet (Tabela 1).

Verificou-se que os alunos possuíam menos contato com a videoconferência do que com a Internet. Além desse aspecto, nenhum aluno possuía experiência de realização de cursos a distância com a utilização dessas duas tecnologias juntas.

\subsection{Perfil dos professores pesquisados}

Os professores apresentaram uma distribuição relativamente homogênea em termos de tempo de docência no ensino superior. Mais de $50 \%$ deles possuíam mais de dez anos de experiência e apenas $20 \%$ possuíam até três anos de experiência em docência.

Esses professores consideraram-se conhecedores dos processos envolvidos na educação a distância. Metade dos professores considerou satisfatório o seu conhecimento sobre EAD, enquanto cerca de $40 \%$ destes avaliaram o seu conhecimento como bom ou ótimo.

\subsection{Resultados da escala atitudinal}

Nas tabelas a seguir, são apresentadas as médias das respostas por assertiva e os respectivos desvios-padrão. A partir da escala utilizada, em que 1) significa pouco, 2) regular, 3) satisfatório, 4) bom e 5) ótimo, as médias mais próximas de 5 referem-se às atitudes mais positivas, e as médias próximas de 1 dizem respeito às atitudes mais negativas. Para se analisar o grau de homogeneidade nas respostas, utilizou-se o desvio-padrão.

\subsubsection{Interação}

A importância da interação na educação a distância tem sido grandemente enfatizada como um meio para aumentar o desempenho e a satisfação do aluno com o curso (Driver, 2002; Moore e Kearsley, 1996). No escopo

Tabela 1. Distribuição de freqüiências do conhecimento dos alunos da videoconferência e da Internet.

\begin{tabular}{lcc}
\hline Experiência com: & $\begin{array}{c}\text { Videoconferência } \\
(\boldsymbol{\%})\end{array}$ & $\begin{array}{c}\text { Internet } \\
(\mathbf{\%})\end{array}$ \\
\hline Inexperiente & 24,97 & 8,17 \\
Iniciante & 56,64 & 39,57 \\
Conhecimento & 18,03 & 48,15 \\
Suficiente & & \\
Expert & 0,34 & 4,08 \\
\hline
\end{tabular}

deste trabalho, interação é compreendida como uma ação recíproca entre duas ou mais pessoas, que pode ser presencial ou mediada por alguma tecnologia.

Conforme pode ser visualizado na Tabela 2, é positiva a atitude de alunos e professores em relação à interação proporcionada pelo modelo entre os alunos e entre os alunos e os professores. O fato dos alunos estarem todos em uma única sala de aula com aparelhos de geração e recepção de videoconferência em tempo real, com um número controlado de alunos (no máximo 30), pode ter influenciado o desenvolvimento dessa atitude. Neste caso, a atitude em relação à dinâmica de interação pode ser interpretada como uma característica geral da classe, em consonância com as pesquisas referentes à interação vicária (Fulford e Zang, 1993). Assim, a visualização da possibilidade real de interação pode ter um impacto maior na atitude dos alunos do que a atitude relacionada à sua participação individual.

Já as atitudes de alunos e professores em relação à interação proporcionada durante o processo de orientação, apresentaram média um pouco menor. Os respondentes consideraram-na regular, com a visão dos professores mais próxima de satisfatório. Uma vez que estes alunos ainda não estavam em fase de orientação de dissertação, as suas atitudes com relação à interação neste período estão mais balizadas pelo afeto do que pela cognição (Fiske e Taylor, 1984), uma vez que as possibilidades interativas do período de orientação de dissertação eram potencialmente as mesmas daquelas do período de créditos.

A diferença de atitudes de professores e alunos quanto a este aspecto também pode estar vinculada a aspectos não relacionados diretamente à educação a distância, e sim aos papéis de orientadores e alunos durante o período de desenvolvimento de uma dissertação. Os alunos tendem a superestimar as necessidades de interação com os orientadores para adquirir segurança para o desenvolvimento de sua dissertação, enquanto os orientadores tendem a minimizá-la (Mullins e Kiley, 2002).

\subsubsection{Corpo docente}

Conforme pode ser visualizado na (Tabela 3), a atitude dos alunos em relação ao corpo docente também foi positiva em todos os aspectos investigados.

Tabela 2. Atitudes de alunos e professores com relação à categoria interação.

\begin{tabular}{|c|c|c|c|c|}
\hline \multirow{2}{*}{$\begin{array}{c}\text { Assertivas } \\
\text { O modelo propicia } \\
\text { interação entre: }\end{array}$} & \multicolumn{2}{|c|}{ Média } & \multicolumn{2}{|c|}{ Desvio-padrão } \\
\hline & Aluno & Prof. & Aluno & Prof. \\
\hline Aluno/aluno & 4,30 & 3,9 & 0,8 & 1,2 \\
\hline Aluno/orient. & 2,32 & 2,7 & 1,3 & 1,1 \\
\hline Aluno/prof. & 3,31 & 3,37 & 1,1 & 1,1 \\
\hline
\end{tabular}


Tabela 3. Atitudes de alunos com relação ao corpo docente

\begin{tabular}{lcc}
\hline \multicolumn{1}{c}{ Corpo Docente } & Média & Desvio-padrão \\
\hline $\begin{array}{l}\text { Tempo de resposta às dúvidas } \\
\text { via ambiente de aprendizagem }\end{array}$ & 3,89 & 1,0 \\
on-line & & \\
$\begin{array}{l}\text { Ambiente de abertura e respeito } \\
\text { Relação entre conteúdo e } \\
\text { realidade }\end{array}$ & 3,21 & 0,8 \\
$\begin{array}{l}\text { Estimula o uso do ambiente de } \\
\text { aprendizagem } \text { on-line }\end{array}$ & 3,46 & 1,1 \\
\hline
\end{tabular}

Esses resultados estão em consonância com os resultados de uma pesquisa, com 334 alunos de graduação que realizaram um curso a distância, conduzida por Inman et al. (1999). Os autores investigaram, entre outros aspectos, a satisfação destes com relação à qualidade do processo de ensino/aprendizagem do curso a distância. Três variáveis foram responsáveis por $69 \%$ da variância nas respostas, entre elas a disponibilidade percebida do professor.

No caso em estudo, verificou-se que os professores apresentaram comportamentos diretos para com os alunos (immediacy behaviors), que dizem respeito a comportamentos comunicativos que reduzem a distância psicológica e social entre as pessoas (Myers et al., 1998). As atitudes positivas diante das assertivas da categoria professor demonstraram os seguintes indícios de comportamentos diretos: manter contato visual; dar e receber feedback, identificado pelas respostas rápidas por parte do professor; relação entre conteúdo e realidade por meio do uso de exemplos reais em sala de aula; etc. Em um estudo com alunos de cursos de $M B A$, Arbaugh (2001) verificou que os comportamentos diretos por parte dos professores e as atitudes dos alunos com relação ao software utilizado no curso foram preditores significativos da satisfação destes com relação ao curso. No presente estudo, os comportamentos diretos por parte dos professores podem estar associados a uma atitude positiva dos alunos ante os seus comportamentos.

\subsubsection{Satisfação das expectativas}

Para finalizar, a questão "De modo geral o curso está atendendo às suas expectativas?", direcionada aos alunos, resultou em uma média de 3,75, com desviopadrão de 0,95 . Verifica-se que as expectativas iniciais dos alunos em relação ao curso foram satisfatoriamente atendidas. Neste estudo, as atitudes positivas dos alunos com relação à interação proporcionada pelo modelo podem ter contribuído positivamente para o sentimento de atendimento de suas expectativas com relação ao curso (Havice, 1999). Esse resultado reforça os resultados encontrados por Valenta et al. (2001), que identificaram que as atitudes de alunos são grandemente afetadas por meio do aumento e da facilitação da interação entre alunos e professores.

A satisfação dos alunos com os seus cursos de mestrado a distância nesta instituição também ratificam os resultados identificados por Dondi et al. (2004) com alunos matriculados em cursos de graduação na comunidade européia. Tanto no estudo europeu quanto neste estudo, as atitudes foram positivas com relação à educação a distância. No caso deste estudo, o temor de que as tecnologias poderiam adicionar um grau de complexidade maior ao processo educacional, de modo a afetar a capacidade de absorção dos alunos, parece não ter ocorrido, mesmo sendo a maioria dos alunos inexperientes ou iniciantes com a videoconferência. Infere-se, então, que, na configuração educacional estudada, as tecnologias não se impuseram ao modelo pedagógico, mas facilitaram as trocas interativas. $\mathrm{O}$ fato de a videoconferência ter sido a principal mídia, por meio da qual cerca de $80 \%$ das aulas foram ministradas, pode ter facilitado o processo, uma vez que ela proporcionou um arranjo educacional muito próximo da experiência tradicional de alunos e professores. Assim, o esquema educacional tradicional/prototípico (ver e falar com o professor em tempo real, os alunos estarem todos presentes em uma "sala de aula") não foi completamente alterado. Sendo a configuração educacional a distância próxima do esquema de representação dos alunos, ela não foi interpretada com apreensão e ceticismo.

\subsection{Atitudes dos alunos e tempo de titulação}

A realização do curso de mestrado acadêmico no Brasil envolve a finalização com sucesso de um número específico de disciplinas, desenvolvimento, registro e aprovação em defesa pública de uma dissertação. O prazo esperado para a realização de um curso de mestrado acadêmico no Brasil pela CAPES/MEC é de até 24 meses. Este prazo é um dos itens levados em consideração pela CAPES/MEC para avaliar a qualidade dos cursos de pós-graduação no país.

Dada a relevância do tempo utilizado pelo aluno para concluir o curso de mestrado e a preocupação atual com a qualidade de cursos a distância (Vidovich e Porter, 1999), procurou-se identificar (Quadro 1) o tempo médio que os alunos levam para alcançar sua titulação nos cursos de mestrado a distância, o tempo médio que os alunos levam para alcançar a titulação dos mesmos cursos presenciais da instituição sob investigação e o tempo médio que os alunos levam para alcançar a titulação dos mesmos cursos presenciais em outras universidades brasileiras. A CAPES atribui, a partir de uma avaliação trienal, um conceito a cada programa de pós-graduação no país. Utilizou-se como parâmetro o tempo médio de titulação dos 
Quadro 1. Tempo médio de titulação em cursos de mestrado em Engenharia de Produção presenciais e a distância no ano de 2000. Fontes: http://ged.capes.gov. br/AgDw/silverstream/pages/frPesquisaColeta.html> e $<$ http://teses.eps.ufsc.br $>$.

\begin{tabular}{lcc}
\hline & Presencial & Distância \\
\hline Tempo médio de titulação & 30 meses & 21 meses \\
no mestrado no curso de & & \\
$\begin{array}{l}\text { Engenharia de Produção } \\
\text { da universidade sob inves- }\end{array}$ & \\
tigação & \\
\hline $\begin{array}{l}\text { Tempo médio de titulação } \\
\text { no mestrado nos cursos de }\end{array}$ & & \\
$\begin{array}{l}\text { Engenharia de Produção } \\
\text { das universidades brasilei- }\end{array}$ & & Não havia \\
ras com o mesmo conceito & & \\
\hline
\end{tabular}

alunos matriculados nos mesmos cursos de mestrado em Engenharia de Produção em outras instituições de ensino superior com o mesmo conceito.

As seções anteriores demonstraram que as atitudes de alunos e professores em relação aos cursos de mestrado em modalidade educacional a distância em Engenharia de Produção foram predominantemente positivas nas categorias analisadas. Ao mesmo tempo, a análise do Quadro 1 nos permite identificar que o tempo médio que o aluno leva para titular-se em cursos de mestrado a distância foi o mais próximo ao ideal de qualidade preconizado pelos órgãos reguladores brasileiros. O tempo de titulação dos alunos a distância foi melhor, tanto em comparação ao dos alunos matriculados nos mesmos cursos presenciais oferecidos pela mesma universidade quanto ao dos alunos matriculados nos mesmos cursos oferecidos por outras universidades brasileiras.

Esses resultados iniciais abrem espaço para a investigação mais detalhada a respeito do papel das atitudes dos alunos diante de cursos de mestrado em Engenharia de Produção (e cursos em geral). Em termos específicos, levantam-se duas hipóteses: 1) que as atitudes positivas podem influenciar no tempo de titulação; e 2) que as atitudes positivas (ou negativas) ante cursos a distância estão relacionadas tanto com o modelo pedagógico e a estrutura de apoio ao aluno quanto com as mídias utilizadas no curso. Assim, propõe-se que os próximos estudos procurem relacionar as atitudes com o modelo educacional subjacente e as mídias utilizadas.

\section{Considerações finais}

O objetivo deste artigo foi o de descrever e analisar parte de um relatório realizado em instituição educacional acerca das atitudes de alunos e professores participantes de curso de mestrado em modalidade educacional a distância, a partir de três aspectos: 1) a interação proporcionada pelo modelo educacional; 2 ) o papel do professor; e 3 ) as expectativas dos alunos pelo curso. $\mathrm{O}$ artigo discutiu, ainda, a influência destas atitudes no tempo de titulação do aluno. Para tanto, o artigo procurou contextualizar teoricamente as atitudes em relação à educação a distância e discutiu a educação a distância a partir da perspectiva da manutenção e/ou do afastamento dos esquemas representativos dos alunos e professores ante a situação prototípica da educação presencial. Neste contexto, ressalta-se a importância do construto dos esquemas representativos para o entendimento das atitudes diante da educação a distância; e, de forma diferenciada dos resultados de McGreal (1994), o artigo apresentou evidências para inferir que as mídias utilizadas como meios didáticos também podem influenciar as atitudes dos alunos em relação aos cursos a distância. Em consonância com os resultados de Valenta et al. (2001), sugere-se que as atitudes dos alunos podem ter sido afetadas positivamente pelo uso da videoconferência, uma vez que ela facilitou as trocas interativas e o desenvolvimento de comportamentos diretos (immediacy behaviors) entre alunos e professores e entre alunos e professores orientadores. Mesmo sendo a maioria dos alunos inexperiente com a videoconferência, ela proporcionou um arranjo educacional muito parecido com o esquema representativo da educação formal tradicional, o que não gerou resistência ou ceticismo por parte dos alunos. Hipotetizou-se, também, que o menor tempo de titulação dos alunos dos cursos de mestrado a distância pode estar relacionado com: 1) as atitudes destes com relação aos aspectos analisados; e 2) o modelo pedagógico e a estrutura de apoio oferecida ao aluno.

Os resultados deste estudo trazem implicações para o desenvolvimento futuro de cursos de mestrado de Engenharia de Produção a distância. Estas se referem principalmente à influência dos esquemas representativos da educação tradicional e a como eles influenciam as atitudes de alunos e professores diante da educação a distância. Ao terem ciência desse aspecto, os dirigentes institucionais poderão delinear programas de preparação para a educação a distância mais eficazes. O artigo também permite sugerir aos desenhistas educacionais que se concentrem prioritariamente na clarificação dos objetivos educacionais dos programas de mestrado e no seu modelo pedagógico como principais estratégias de sucesso. As mídias utilizadas também são importantes, principalmente se forem levadas em consideração as possibilidades pedagógicas e interativas de cada uma (videoconferência e internet) no que diz respeito ao esquema vigente de educação formal tradicional.

Em termos de limitações, ressalta-se que este estudo é de natureza exploratória e, como tal, não pode ser comple- 
tamente generalizado para outros ambientes e populações de alunos e professores. Além deste aspecto, o instrumento de levantamento de dados, apesar de ter sido elaborado a partir de escalas consolidadas, precisa de validação estatística, de modo que se tenha certeza de que ele mede e operacionaliza efetivamente os construtos sob investigação. Por fim, os resultados do estudo refletem tendências iniciais e poderão ser mais detalhadamente investigados em estudos futuros. Neste sentido, o presente estudo se posiciona, de acordo com Snow e Thomas (1994), com o foco na identificação de insumos para a construção de teorias, e não em sua testagem per se.

\section{Agradecimentos}

Os autores gostariam de agradecer a Dulce Márcia Cruz e Luciana Saraiva, pelas importantes contribuições em versões anteriores deste artigo, e a Nayara Gondim e Janae Gonçalves Martins, pelo auxílio nos cálculos estatísticos.
AJZEN, I. Nature and operations of attitudes. Annual Reviews Psychology, v. 52, p. 27-58, 2001

AKERLING, G.; TREVITT, C. Enhancing self-directed learning through educational technology: when students resist the change. Innovations in Education and Training International, v. 36, n. 2, p. 96-105, 1999.

ARBAUGH, J. B. How Instructor immediacy behaviors affect student satisfaction and learning in web-based courses. Business Communication Quarterly, v. 64, n. 4, p. 42-54, 2001.

BARGH, J. A.; CHARTRAND, T. L. The unbearable automaticity of being. American Psychologist, v. 54, p. 462-479, 1999.

BENNET, R.; KOTTASZ, R. Marketing undergraduates' attitudes towards query-based instructional machines as possible learning medium. British Journal of Educational Technology, v. 32, n. 4, p. 471-482, 2001.

BERGE, Z. L. Obstacles do distance training and education in corporate organizations. Journal of Workplace Learning, v. 14, n. 5, p. 182-189, 2002.

DONDI, C., et al. Percepciones de los estudiantes sobre la relevancia de las TIC para estudios universitarios. In: SANGRÀ, A.; SANMAMED, M. G. (Coord.). La transformación de las universidades a través de las TIC: discursos y prácticas. Barcelona: Editorial OUC, 2004. p. 99-109.

DRIVER, M. Exploring student perceptions of group interaction and class satisfaction in the web-enhanced classroom. Internet and Higher Education, v. 5, p. 35-45, 2002.

FISKE, S. T.; TAYLOR, S. E. Social cognition. New York: Random House, 1984.

FULFORD, C. P.; ZHANG, S. Perceptions of interaction: the critical predictor in distance education. American Journal of Distance Education, v. 7, n. 3, p. 8-21, 1993.
HAVICE, W. L. College students' attitudes toward oral lectures and integrated media presentations. The Journal of Technology Studies, v. 25, n. 1, p. 51-55, 1999.

INMAN, E., et al. Instructor and student attitudes toward distance learning. Community College Journal of Research and Practice, v. 23, p. 581-591, 1999.

LAVINE, H., et al. The primacy of affect in the determination of attitudes and behavior: the moderating role of affective-cognitive ambivalence. Journal of Experimental Social Psychology, v. 34, p. 398-421, 1998.

MCGREAL, R. Comparison of the attitudes of learners taking audio graphic teleconferencing courses in secondary schools in northern Ontario. Interpersonal Computing and Technology, v. 2, n. 4, p. 11-23, 1994.

MOORE, M. G.; KEARSLEY, G. Distance education: a systems view. California: Wadsworth Publishing, 1996.

MULLINS, G.; KILEY, M. 'It's a PhD, not a Nobel Prize`: how experienced examiners assess research theses. Studies in Higher Education, v. 27, n. 4, p. 369-386, 2002.

MYERS, S. A., et al. Instructor immediacy in the Chinese college classroom. Communication Studies, v. 49, p. 240-253, 1998.

OCKER, R. J. Collaborative learning environments: exploring students' attitudes and satisfaction in face-toface and asynchronous computer conferencing settings. Journal of Interactive Learning Research, v. 12, n. 4, p. 427-448, 2001.

PEREZ, E. C.; WHITE, M. A. Student evaluation of motivational and learning attributes of microcomputer software. Journal of Computer Based Instruction, v. 12, n. 2, p. 39-43, 1985.

PHIPPS, R.; MERISOTIS, J. What's the difference? A review of contemporary research on the effectiveness of distance learning in higher education. American Federation of Teachers; National Education Association; The Institute for Higher Education Policy. Washington, 1999. 
SINGHANAYAK, C.; HOOPER, S. The effects of cooperative learning and learner control on students' achievement, option selections, and attitudes. Educational Technology Research and Development, v. 46, n. 2, p. 17-33, 1998.

SNOW, C.; THOMAS, J. Field research methods in strategic management: contributions to theory building and testing. Journal of Management Studies, v. 31, n. 4, p. 457-480, 1994.

UNIVERSIDADE FEDERAL DE SANTA CATARINA. Laboratório de Ensino a Distância. Modelo pedagógi- co da pós-graduação presencial virtual do PPGEP/ UFSC. Florianópolis, 2001. p. 76.

VALENTA, A. et al. Identifying student attitudes and learning styles in distance education. Journal of Asynchronous Learning Networks, v. 5, n. 2, p. 111-127, 2001.

VIDOVICH, L.; PORTER, P. Quality policy in Australian higher education of the 1990s: University perspectives. Journal of Educational Policy, v. 14, n. 6, p. 567-586, 1999.

WOOD, W. Attitude change: persuasion and social influence. Annual Reviews, v. 51, p. 539-570, 2000.

\title{
STUDENT AND TEACHER ATTITUDES REGARDING MASTER'S DISTANCE LEARNING PROGRAMS IN PRODUCTION ENGINEERING
}

\begin{abstract}
This paper analyses the reported results of an evaluation of the development process of master's level post-graduate distance education courses in Production Engineering. This initiative, the only one of its kind in Brazil, won a Quality Award from the Brazilian Distance Learning Association (ABED). This analysis involved three aspects evaluated in the report with respect to teachers' and students' attitudes toward distance learning: (A) teachers' and students' attitudes toward the quality of interaction; (B) students' attitudes toward the teacher's role; and $(C)$ students' expectations regarding the course. The theoretical approach was the theory of attitudes, the concept of representative schemata, and the constructs relating to attitudes about different aspects of distance learning. This is an exploratory, quantitative study which analyzes the attitudes of teachers and students involved in a master's course in Production Engineering in a distance learning setting. We discuss how the students' attitudes influence the time they take to complete the course. The subjects of this survey are 471 students and 30 teachers. The data was collected from a questionnaire. Our findings suggest that the students' and teachers' attitudes regarding the aspects investigated were predominantly positive. Time to completion was about two years, which is less than the time consumed for a similar, traditional master's program offered by the same institution. Unlike the findings of some surveys on the role of attitudes toward distance learning, the results of our study suggest that positive (or negative) attitudes toward distance learning involve both the pedagogical model and the technology employed. These findings beg a more detailed investigation about the role of students' attitudes in master's programs in Production Engineering.
\end{abstract}

Keywords: distance education, attitudes, production engineering, post-graduate education. 
